\title{
Predictive factors of symptomatic lumbar canal stenosis in patients after surgery for cervical spondylotic myelopathy
}

This article was published in the following Dove Press journal:

Therapeutics and Clinical Risk Management

\author{
Lingde Kong* \\ Jiangbo Bai* \\ Bing Zhang \\ Yong Shen \\ Dehu Tian
}

Department of Orthopedics, The Third Hospital of Hebei Medical University, Shijiazhuang, Hebei,

People's Republic of China

*These authors contributed equally to this work
Correspondence: Dehu Tian

Department of Orthopedics, The Third Hospital of Hebei Medical University, 139 Ziqiang Road, Shijiazhuang, Hebei 05005I, People's Republic of China

Tel +86 3II 88602307

Email tiandhhand@qq.com
Background: The aim of this study was to determine the incidence of coexisting symptomatic lumbar canal stenosis (LCS) in patients after surgery for cervical spondylotic myelopathy (CSM) and identify possible predictive factors associated with it.

Materials and methods: We retrospectively reviewed patients with CSM at our institution between January 2005 and December 2015. Clinical and radiographic factors including age, gender, body mass index, Japanese Orthopedic Association scores, cervical alignment, anteroposterior diameter of cervical canal, number of levels with CSM, and percentage of cervical cord compression were investigated. Symptomatic LCS was defined as leg symptoms and a narrowing of the lumbar spinal canal at one level at least, which is confirmed by magnetic resonance images of the lumbar spine. Univariate and multivariate analyses were used to identify possible predictive factors. Pearson correlation analysis was also conducted to analyze the association between cervical parameters and percentage of LCS.

Results: A total of 317 patients with CSM met the criteria for inclusion. There were 39 patients $(12.3 \%)$ with LCS after cervical surgery during a mean of 7.3 years' follow-up. In the multivariate logistic regression analysis, narrow diameter of cervical canal (OR, 3.96; 95\% CI, 1.20-13.04) was identified as the only independent predictor of symptomatic LCS in CSM patients. The correlation coefficient between anteroposterior diameter of cervical canal and severity of LCS indicated a significantly positive linear relationship with $0.536(P<0.001)$.

Conclusion: We found that narrow diameter of cervical canal was associated with symptomatic LCS in CSM patients. Patients with this risk factor should be informed the possibility of symptomatic LCS.

Keywords: predictive factors, lumbar canal stenosis, cervical spondylotic myelopathy, MR imaging

\section{Introduction}

Spinal stenosis, which is caused by the progressive narrowing of the spinal canal through a degenerative process, is a common disease in elderly patients. ${ }^{1,2}$ As we know, spinal stenosis can occur at any level, but it was frequently seen at the segments with great mobility, such as cervical or lumbar spine. Spondylosis affects not just a single segment of the spine, it is generally more widespread. Thus, coexisting stenosis at different anatomical segments is frequently seen. ${ }^{3-5}$ The overall degenerative changes in the cervical spine could accompany concurrent degenerative changes in the lumbar spine. In 1964, Teng and Papatheodorou ${ }^{6}$ described the concurrent cervical and lumbar stenosis at the first time. Dagi et $\mathrm{al}^{7}$ used the term "tandem spinal stenosis" to describe 
combined stenosis at the year of 1987. Concurrent stenosis could add extra difficulty in diagnosis and treatment to orthopedic surgeons.

Cervical spondylotic myelopathy (CSM) patients show various symptoms, such as clumsiness of the hands, spastic gait, loss of balance or sensory impairment, ${ }^{8,9}$ while the major clinical diagnostic feature of lumbar canal stenosis (LCS) is intermittent claudication. ${ }^{10,11}$ As cervical myelopathy can also cause disturbances of gait or difficulty in standing, the symptoms and signs indicating cervical myelopathy may make it difficult to distinguish the LCS-related symptoms. At the same time, imaging of the lumbar spine is not routinely performed in patients with cervical myelopathy, latent stenosis of lumbar canal is frequently neglected. In some cases, symptoms of LCS are diagnosed by mistake as a result of poor clinical outcomes following cervical myelopathy surgeries. It is therefore essential for us to have a good knowledge of the symptomatic LCS following cervical spine surgeries.

In this study, we reviewed CSM patients after surgery and analyzed related data of patients with the possible symptoms of LCS. The aim was to determine the incidence of coexisting symptomatic LCS in patients after surgery for CSM. In addition, possible predictive factors associated with symptomatic LCS were also sought.

\section{Materials and methods Patient population}

We retrospectively reviewed CSM patients at our institution between January 2005 and December 2015. The inclusion criteria were adult patients with single- or multiple-level CSM confirmed by correlating magnetic resonance (MR) images. Those with nondegenerative disease, such as trauma, infection, tumor, deformity, or inflammation, or undergoing any prior spinal surgery were excluded. Those showing ossification of posterior longitudinal ligament or thoracic canal stenosis based on computed tomography and MR images were also excluded. This study was approved by the Regional Ethics Committee of the Third Hospital of Hebei Medical University. All the clinical data were collected after acquisition of written informed consent from the patients.

\section{Treatment and follow-up}

Before surgery, all patients underwent plain radiographs, computed tomography, as well as MR imaging test. Anterior cervical decompression and fusion or laminoplasty procedures were performed for these patients. After surgery, patients were followed up postoperatively at 6 weeks, 3, 6, and 12 months, and then annually. Cases that developed symptoms of LCS were advised to go back to clinic at their earliest convenience, and MR imaging was performed for confirmation and further evaluation.

Symptomatic LCS was defined as leg symptoms that were elicited while walking and standing and relieved by forward flexion of the spine or by squatting, and a narrowing of the lumbar spinal canal at one level at least, which is confirmed by MR images of the lumbar spine. Asymptomatic LCS was not analyzed in our study.

\section{Data collection}

In order to analyze the predictive factors, relevant clinical factors including age, gender, body mass index (BMI), and Japanese Orthopedic Association (JOA) scores ${ }^{12}$ were collected from the records of the patients. Preoperative radiographs of cervical spine yielded imaging parameters as follows: from standing lateral radiographs, the Cobb angle from $\mathrm{C} 2$ to $\mathrm{C} 7$ was measured. An alignment of C2-C7 Cobb angle more than 0 was defined as lordosis, and an alignment of $\mathrm{C} 2-\mathrm{C} 7 \mathrm{Cobb}$ angle of 0 or less was defined as kyphosis. ${ }^{13}$ From MR images of cervical spine, the anteroposterior (AP) diameter of cervical canal were measured at the mid-vertebra level on T2 sagittal MR images from C4 to C7, and the mean value was calculated. ${ }^{12}$ The extent of spinal cord compression was defined by the ratio of the spinal cord diameter of the narrowest part to that of the $\mathrm{C} 2 / \mathrm{C} 3$ intervertebral level using sagittal images on T2-weighted MR images (Figure 1). Besides, the number of compression-affected levels on T2-weighted MR images was also counted. Levels showing compress lesions of the spinal cord with or without signal changes were considered compression affected.

From MR images of the lumbar spine in patients with LCS, the percentage of LCS was calculated by a ratio of the mid-sagittal spinal canal diameter at the level of the intervertebral disc to the spinal canal diameter at the mid-vertebra level of the upper vertebral body. The average ratio of each level on T2 sagittal MR images from L2 to S1 was calculated (Figure 2). Besides, the MR imaging classification system recommended by van Eck et al ${ }^{14}$ for lumbar spinal stenosis was used to classify these patients (Table 1). Data measurements were performed by two independent authors, and the mean value was used for analysis.

\section{Statistical analysis}

Descriptive analysis of the patient population was conducted using means and SD for continuous variables and frequencies and percentages for categorical variables. Kaplan-Meier analysis was used to calculate disease-free 


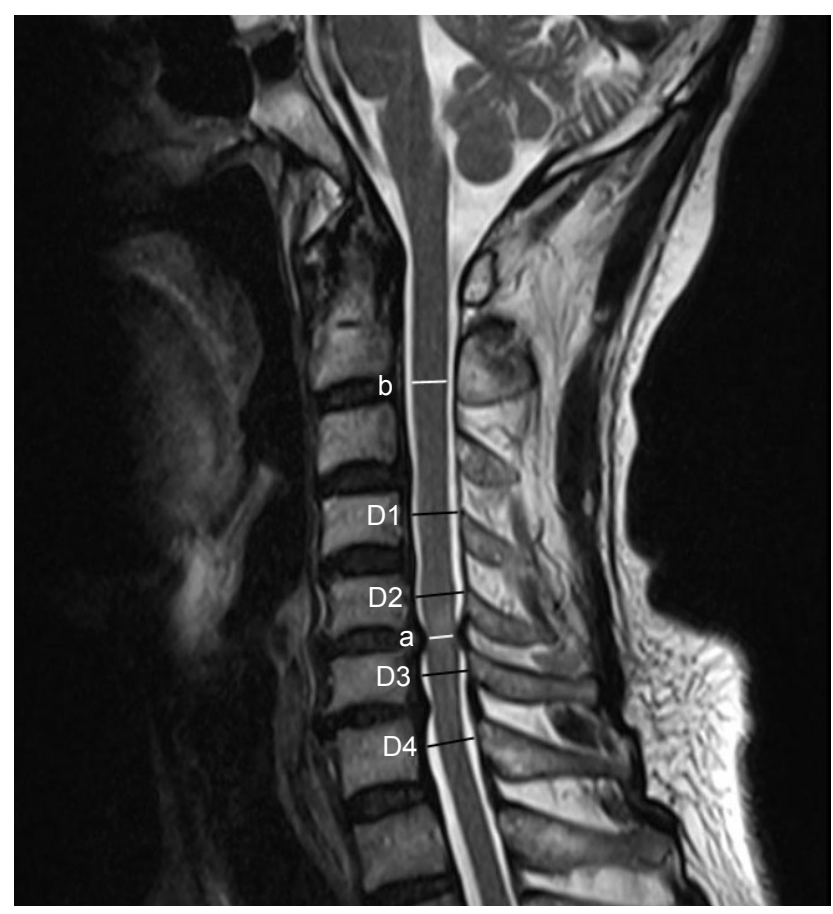

Figure I Measurement of AP diameter of cervical canal and spinal cord compression on $T 2$ sagittal $M R$ imaging.

Notes: DI-D4 were diameters of cervical canal at the mid-vertebra level from C4 to C7. AP diameter of cervical canal $(\mathrm{mm})=(\mathrm{DI}+\mathrm{D} 2+\mathrm{D} 3+\mathrm{D} 4) / 4$. $\mathrm{a}$ and $\mathrm{b}$ are the spinal cord diameters of narrowest part and the $\mathrm{C} 2 / \mathrm{C} 3$ intervertebral level. Spinal cord compression $(\%)=\mathrm{a} / \mathrm{b}$.

Abbreviations: AP, anteroposterior; MR, magnetic resonance.

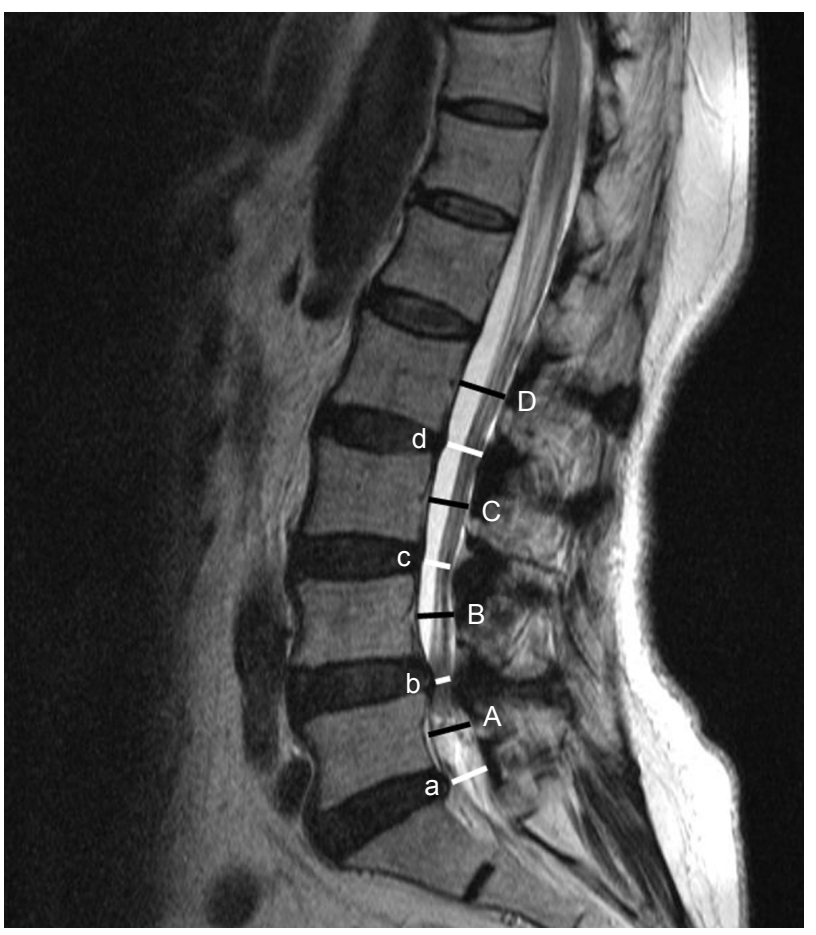

Figure 2 The lumbar canal was calculated by a ratio of the mid-sagittal spinal canal diameter at the level of the intervertebral disc to the spinal canal diameter at the mid-vertebra level of the upper vertebral body.

Note: The percentage of lumbar canal stenosis $(\%)=(a / A+b / B+c / C+d / D) / 4$.
Table I Classification system for lumbar spinal stenosis based on MR images

\begin{tabular}{|c|c|}
\hline Type I & Normal spinal canal \\
\hline Type lla & $\begin{array}{l}\text { Tapering of the spinal canal with gradual narrowing from the } \\
\text { thoracolumbar junction to a peak area of stenosis at L5-SI }\end{array}$ \\
\hline Type llb & $\begin{array}{l}\text { Hourglass stenosis with a canal that begins to narrow at the } \\
\text { thoracolumbar junction down to a peak area of stenosis, } \\
\text { typically at the L3-L4 level, and then widens again caudally }\end{array}$ \\
\hline Type III & $\begin{array}{l}\text { Global stenosis with a symmetrically narrow canal } \\
\text { throughout all lumbar segments with little to no spinal fluid } \\
\text { surrounding the conus }\end{array}$ \\
\hline
\end{tabular}

Abbreviation: MR, magnetic resonance.

survival rate for symptomatic LCS. Fisher exact tests or independent samples $t$-tests were used to determine the difference between groups. All variables found to be potentially associated with LCS $(P<0.10)$ were entered into the multiple logistic regression analysis to analyze their relative importance. Furthermore, Pearson correlation analysis was conducted to analyze the association between cervical parameters and percentage of LCS. A $P$-value $<0.05$ was considered statistically significant. Data analyses were performed using SPSS version 18 for Windows (SPSS, Inc., Chicago, IL, USA).

\section{Results}

A total of 317 patients with CSM met the criteria for inclusion. There were 208 males $(65.6 \%)$ and 109 females (34.4\%). The age of these patients were $63.9 \pm 7.6$ years. One hundred and sixty-six patients (52.4\%) underwent 1-level surgery, 110 (34.7\%) underwent 2-level surgery, 34 (10.7\%) underwent 3-level surgery, and 7 (2.2\%) underwent 4-level surgery. Two hundred and eighty-six procedures were anterior cervical discectomy and fusion, and the other 31 were laminoplasty.

Patients were followed up for $7.3 \pm 4.1$ years. There were 39 patients (12.3\%) with LCS after cervical surgery. Twentytwo were males and 17 were females. Of the 39 patients, 7 patients were detected before cervical surgery, and the other 32 patients were diagnosed during follow-up. KaplanMeier analysis showed a $90.4 \%$ disease-free survival rate for symptomatic LCS (95\% CI, 86.9\%-94.0\%) at 5 years and $83.1 \%$ (95\% CI, $77.8 \%-88.4 \%$ ) at 10 years (Figure 3). The responsible segments of symptomatic LCS were found most commonly at the L4/L5 level, and it was followed by the L5/S1, L3/L4, and L2/L3 levels in descending order of frequency. The numbers of patients with type IIa, type IIb, and type III LCS were 13 (33.3\%), 7 (17.9\%), and 19 (48.7\%), respectively. Among them, 31 patients with symptomatic 


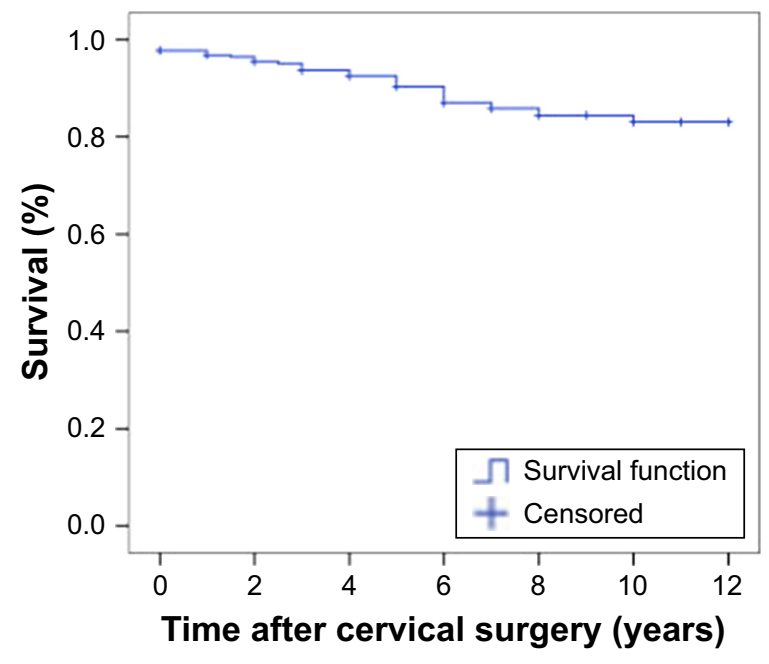

Figure 3 Kaplan-Meier survivorship curve for symptomatic LCS after CSM surgery. Abbreviations: CSM, cervical spondylotic myelopathy; LCS, lumbar canal stenosis.

LCS underwent posterior lumbar interbody fusion, whereas the other 8 patients refused surgery and accepted conventional treatment.

In the univariate analysis, AP diameter of cervical canal in LCS patients was $12.7 \pm 1.4 \mathrm{~mm}$, and was significantly lower than that in non-LCS patients $(P<0.001)$, which was $14.9 \pm 1.6 \mathrm{~mm}$. Multiple-level CSM was disclosed by MR imaging in 26 patients (66.7\%) with LCS and in 125 $(45.0 \%)$ non-LCS patients. The difference is highly statistically significant $(P=0.016)$. However, there were no significant differences in age, gender, BMI, preoperative JOA score, sagittal alignment, and percentage of cervical cord compression between symptomatic LCS and non-LCS patients. The details of result are listed in Table 2 . In the

Table 2 Patients' demographic and baseline data for two groups

\begin{tabular}{|c|c|c|c|}
\hline Variables & $\begin{array}{l}\text { LCS } \\
\text { patients } \\
(\mathbf{N}=39)\end{array}$ & $\begin{array}{l}\text { Non-LCS } \\
\text { patients } \\
(\mathbf{N}=\mathbf{2 7 8})\end{array}$ & P-value \\
\hline Age (years) & $65.1 \pm 8.6$ & $63.7 \pm 7.0$ & 0.195 \\
\hline \multicolumn{4}{|l|}{ Gender } \\
\hline Male & 22 & 186 & 0.211 \\
\hline Female & 17 & 92 & \\
\hline BMI $\left(\mathrm{kg} / \mathrm{m}^{2}\right)$ & $24.8 \pm 6.1$ & $25.9 \pm 6.7$ & 0.332 \\
\hline Preoperative JOA score (points) & $9.5 \pm 2.5$ & $9.8 \pm 2.2$ & 0.434 \\
\hline \multicolumn{4}{|l|}{ Sagittal alignment } \\
\hline Kyphosis & 6 & 34 & 0.606 \\
\hline Lordosis & 33 & 244 & \\
\hline \multicolumn{4}{|l|}{ Levels with CSM } \\
\hline Single level & 13 & 153 & 0.016 \\
\hline Multiple level & 26 & 125 & \\
\hline AP diameter of cervical canal $(\mathrm{mm})$ & $12.7 \pm 1.4$ & $14.9 \pm 1.6$ & $<0.001$ \\
\hline Cervical cord compression (\%) & $76.3 \pm 8.1$ & $78.2 \pm 6.9$ & 0.117 \\
\hline
\end{tabular}

Abbreviations: AP, anteroposterior; BMI, body mass index; CSM, cervical spondylotic myelopathy; JOA, Japanese Orthopedic Association; LCS, lumbar canal stenosis.

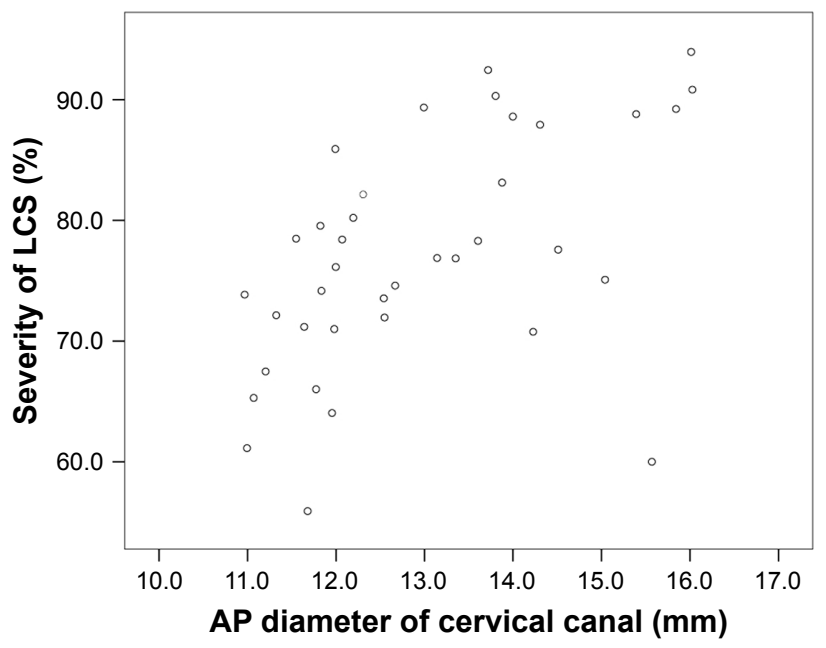

Figure 4 Scatter diagram showing the relationship between AP diameter of cervical canal and severity of LCS.

Abbreviations: AP, anteroposterior; LCS, lumbar canal stenosis.

multivariate logistic regression analysis, narrow diameter of cervical canal (OR, 3.96; 95\% CI, 1.20-13.04) was identified as the only independent predictor of symptomatic LCS in CSM patients. Other parameters were not demonstrated to be associated factors.

Of the 39 patients with symptomatic LCS, we further performed Pearson correlation analysis to analyze the association between cervical cord compression as well as AP diameter of cervical canal and severity of LCS. The correlation coefficient between the cervical cord compression and the severity of LCS was 0.410 , revealing a nonsignificant linear relationship $(P=0.43)$. However, the correlation coefficient between AP diameter of cervical canal and severity of LCS indicated a significantly positive linear relationship with $0.536(P<0.001)$ (Figure 4).

\section{Discussion}

In this study, we reviewed 317 patients surgically treated for CSM and revealed that the prevalence of LCS was $12.3 \%$ with a mean of 7.3 years follow-up. Patients with narrow AP diameter of cervical canal have a higher risk of development of symptomatic LCS after CSM surgeries. AP diameter of cervical canal on MR images was associated with the severity of LCS positively. Therefore, clinical suspicion and early diagnosis are required for CSM patients with narrow canal of cervical spine as they have a higher risk of developing symptomatic LCS later.

The incidence of simultaneous stenosis of the cervical and lumbar spine has been documented by previous studies, but corresponding information varies widely. In a cadaveric study of the general population performed by Lee et al, ${ }^{15}$ the prevalence of tandem stenosis ranged from $0.9 \%$ to 
$5.4 \%$, but in the study by Bajwa et $a 1,{ }^{4}$ the prevalence was $2.05 \%$. Adamova et $\mathrm{al}^{16}$ performed a cross-sectional study of 78 patients with LCS, and found $16.7 \%$ of these patients have clinically symptomatic CSM. In the study by Tsutsumimoto et al, ${ }^{17}$ of 214 patients who had undergone cervical laminoplasty for cervical myelopathy, they identified $13 \%$ patients with symptomatic LCS. We assumed that the different prevalence mainly depends on the group of patients or population being evaluated and on definitions of radiological criteria.

To evaluate the predictive factors associated with the incidence of concurrent lumbar lesions, we analyzed age, gender, BMI, number of CSM levels, AP diameter of cervical canal, and other factors. Lee et $\mathrm{al}^{18}$ noted that those lumbar stenosis patients who are older, male, and have disease involving multiple segments are more likely to develop cervical cord compression in a more severe extent. In the analysis of CSM patients in our study, we did not confirm those factors that were associated with the occurrence of symptom LCS. Only AP diameter of cervical canal showed a significant correlation with the occurrence of LCS. We think patients with developmental narrow canal of cervical spine have a high risk of developing neurologic symptoms due to LCS. This result was in accordance with a conclusion from a previous study by Iizuka et al, ${ }^{19}$ in which they used the TorgPavlov ratio to evaluate cervical spinal stenosis and found the ratio was a predictive factor for CSM in patients with LCS. It seems that LCS or CSM patients with developmental spinal stenosis and are prone to develop symptoms of degenerative diseases. If CSM patients showed narrow canal of cervical spine, extra caution should be taken during the process of follow-up, and early MR imaging examination of the lumbar spine is recommended to reveal the compressive lesions of nerve system.

Cervical canal stenosis was usually evaluated by the AP diameter of the cervical spinal canal on plain lateral radiographs, but this measurement includes a magnification error resulting from the focus-to-film distance and the object-to-film distance. The ratio of the sagittal diameter of the cervical spinal canal to that of the vertebral body was developed by Torg and Pavlov as an indicator of the degree of developmental spinal canal narrowing. ${ }^{20}$ Although the Torg-Pavlov ratio excludes variable enlargement factors, it failed to consider the impact of soft tissue on CSM, including hypertrophy of the ligamentum flavum. ${ }^{12}$ We consider the diameter of the cerebrospinal fluid column on MR images to more accurately reflect the space in the cervical canal. In this study, AP diameter of cervical canal and severity of LCS indicated a positive linear relationship. van Eck et al $^{14}$ develop a simple and clinical useful morphological classification system for congenital lumbar spinal stenosis allowing clinicians to recognize patterns of lumbar congenital stenosis quickly and be able to screen these patients for tandem cervical stenosis. We used this system in our study to classify patients with tandem spinal stenosis. The results showed that $48.7 \%$ patients were type III LCS, which is characterized by global stenosis with a symmetrically narrow canal throughout all lumbar segments. We assumed that this type of LCS may be associated with tandem spinal stenosis. However, further studies based on a large population are still required to confirm this conclusion.

This study has several limitations. First, the extent of spinal stenosis was only measured by mid-sagittal images. Some patients may have more severe stenosis at paracentral area of spinal canal. If a new stenosis index, involving both sagittal and axial images, can be used for the measurement of stenosis by cross-sectional area, the results might be more precise than the current study. Besides, only a limited numbers of predictive factors were investigated in our study; the involvement of other factors in further study may provide more information to us.

\section{Conclusion}

In summary, we found that the prevalence of LCS was $12.3 \%$ in patients following surgery for CSM. Patients with narrow canal of the cervical spine have a higher risk of development of symptomatic LCS after CSM surgeries. AP diameter of cervical canal on MR images was associated with the severity of LCS. We recommended that clinical suspicion and early diagnosis are especially required for CSM patients with narrow canal of cervical spine as they have a higher risk of developing into symptomatic LCS later.

\section{Acknowledgment}

We are grateful to Dr Bin Zhang for his assistance in the statistical analysis.

\section{Disclosure}

The authors report no conflicts of interest in this work.

\section{References}

1. Harrop JS, Hilibrand A, Mihalovich KE, Dettori JR, Chapman J. Costeffectiveness of surgical treatment for degenerative spondylolisthesis and spinal stenosis. Spine (Phila Pa 1976). 2014;39:S75-S85.

2. Tosteson AN, Lurie JD, Tosteson TD, et al. Surgical treatment of spinal stenosis with and without degenerative spondylolisthesis: costeffectiveness after 2 years. Ann Intern Med. 2008;149:845-853.

3. Laban MM, Green ML. Concurrent (tandem) cervical and lumbar spinal stenosis: a 10-yr review of 54 hospitalized patients. Am J Phys Med Rehabil. 2004;83:187-190. 
4. Bajwa NS, Toy JO, Young EY, Ahn NU. Is congenital bony stenosis of the cervical spine associated with lumbar spine stenosis? An anatomical study of 1072 human cadaveric specimens. J Neurosurg Spine. 2012; 17:24-29.

5. Park MS, Moon SH, Kim TH, et al. Asymptomatic stenosis in the cervical and thoracic spines of patients with symptomatic lumbar stenosis. Global Spine J. 2015;5:366-371.

6. Teng P, Papatheodorou C. Combined cervical and lumbar spondylosis. Arch Neurol. 1964;10:298-307.

7. Dagi TF, Tarkington MA, Leech JJ. Tandem lumbar and cervical spinal stenosis. Natural history, prognostic indices, and results after surgical decompression. J Neurosurg. 1987;66:842-849.

8. Rao RD, Gourab K, David KS. Operative treatment of cervical spondylotic myelopathy. J Bone Joint Surg Am. 2006;88:1619-1640.

9. Singh A, Crockard HA. Quantitative assessment of cervical spondylotic myelopathy by a simple walking test. Lancet. 1999;354:370-373.

10. Weinstein JN, Tosteson TD, Lurie JD, et al. Surgical versus nonsurgical therapy for lumbar spinal stenosis. N Engl J Med. 2008;358: 794-810.

11. Kim YU, Kong YG, Lee J, et al. Clinical symptoms of lumbar spinal stenosis associated with morphological parameters on magnetic resonance images. Eur Spine J. 2015;24:2236-2243.

12. Kong LD, Meng LC, Wang LF, Shen Y, Wang P, Shang ZK. Evaluation of conservative treatment and timing of surgical intervention for mild forms of cervical spondylotic myelopathy. Exp Ther Med. 2013;6: $852-856$.
13. Zhang JT, Li JQ, Niu RJ, Liu Z, Tong T, Shen Y. Predictors of cervical lordosis loss after laminoplasty in patients with cervical spondylotic myelopathy. Eur Spine J. 2017;26:1205-1210.

14. van Eck CF, Spina IN, Lee JY. A novel MRI classification system for congenital functional lumbar spinal stenosis predicts the risk for tandem cervical spinal stenosis. Eur Spine J. 2017;26:368-373.

15. Lee MJ, Garcia R, Cassinelli EH, Furey C, Riew KD. Tandem stenosis: a cadaveric study in osseous morphology. Spine J. 2008;8:1003-1006.

16. Adamova B, Bednarik J, Andrasinova T, et al. Does lumbar spinal stenosis increase the risk of spondylotic cervical spinal cord compression? Eur Spine J. 2015;24:2946-2953.

17. Tsutsumimoto T, Shimogata M, Yui M, Ohta H, Misawa H. The natural history of asymptomatic lumbar canal stenosis in patients undergoing surgery for cervical myelopathy. J Bone Joint Surg Br. 2012;94: 378-384.

18. Lee SH, Kim KT, Suk KS, et al. Asymptomatic cervical cord compression in lumbar spinal stenosis patients: a whole spine magnetic resonance imaging study. Spine (Phila Pa 1976). 2010;35:2057-2063.

19. Iizuka H, Takahashi K, Tanaka S, Kawamura K, Okano Y, Oda H. Predictive factors of cervical spondylotic myelopathy in patients with lumbar spinal stenosis. Arch Orthop Trauma Surg. 2012;132:607-611.

20. Pavlov H, Torg JS, Robie B, Jahre C. Cervical spinal stenosis: determination with vertebral body ratio method. Radiology. 1987;164: $771-775$.
Therapeutics and Clinical Risk Management

\section{Publish your work in this journal}

Therapeutics and Clinical Risk Management is an international, peerreviewed journal of clinical therapeutics and risk management, focusing on concise rapid reporting of clinical studies in all therapeutic areas, outcomes, safety, and programs for the effective, safe, and sustained use of medicines. This journal is indexed on PubMed Central, CAS,

\section{Dovepress}

EMBase, Scopus and the Elsevier Bibliographic databases. The manuscript management system is completely online and includes a very quick and fair peer-review system, which is all easy to use. Visit http://www.dovepress.com/testimonials.php to read real quotes from published authors. 